\title{
A low-dimension structure strategy for flexible photodetectors based on perovskite nanosheets/ZnO nanowires with broadband photoresponse
}

\author{
Shalong Wang, Zhengfeng Zhu, Yousheng Zou ${ }^{*}$, Yuhang Dong, Shuting Liu, Jie Xue, Leimeng Xu, \\ Yuhui Dong and Jizhong Song
}

\begin{abstract}
Flexible photodetectors (PDs) have huge potential for application in next-generation optoelectronic devices due to their lightweight design, portability, and excellent large area compatibility. The main challenge in the construction of flexible PDs is to maintain the optoelectronic performance during repetitive bending, folding and stretching. Herein, flexible PDs based on $\mathrm{ZnO}$ nanowires (NWs) and $\mathrm{CsPbBr}_{3}$ nanosheets (NSs) were constructed by an integrated low-dimensional structure strategy. Benefiting from the flexibility of unique sheet and wire structures, the PDs were able to maintain excellent operational stability under various mechanical stresses. For example, the PDs exhibited no obvious changes in optoelectronic performance after bending for 1000 times. Additionally, the PDs exhibited an integrated broadband response ranging from ultraviolet to visible region due to the combination of the intrinsic light absorption capability of $\mathrm{ZnO}$ and $\mathrm{CsPbBr}_{3}$. The PDs demonstrated high responsivities of 3.10 and $0.97 \mathrm{~A} \mathrm{~W} \mathrm{~W}^{-1}$ and detectivities of $5.57 \times 10^{12}$ and $1.71 \times 10^{12}$ Jones under ultraviolet and visible light irradiation, respectively. The proposed construction strategy for highly flexible and performance-integrated PDs shows great potential in future smart, wearable optoelectronic devices.
\end{abstract}

Keywords: perovskite, $\mathrm{ZnO}$ nanowires, flexible photodetectors, broad spectrum

\section{INTRODUCTION}

Flexible electronic devices with highly integrated, compact, and portable features are very attractive in the fields of smart photodetectors (PDs) [1-7], wearable sensors [8-11], bendable solar cells [12-15], and field-emission transistors [16-19]. Among various flexible devices, PDs have aroused extensive attention due to their wide ap- plications in flame detection, optical communication, and environmental monitoring [20-25]. In general, flexible PDs are fabricated on bendable or ductile substrates (such as plastic, metal sheets, and rubber), which requires the selected semiconductor materials possessing the ability to operate well while bent, rolled or stretched. Thus, developing suitable semiconductor materials for flexible PDs is highly desirable.

Generally, thin-film materials and low-dimensional materials are employed for the construction of flexible PDs. However, the optoelectronic performance of thin film-based devices may deteriorate because of the structural damage caused by sustained stretching stress. In addition, cracks can easily extend from the top to the bottom of the film under mechanical stress, which will block the carrier transport and eventually lead to degradation, even the expiration of the PDs [26-28]. Whereas, cracking in low-dimensional materials can be prevented because the generation potential of crack decreases linearly with decreasing dimensions. Furthermore, materials with low-dimensional nanostructures have much higher photo-sensitivity and faster carrier transport. Consequently, low-dimensional materials with excellent optical and electrical properties possess broad prospect in flexible PDs.

Among the various low-dimensional materials, the zero dimensional (0D) nanoparticle-based film is similar to bulk film, which also suffers from cracks under repeated mechanical stresses. Therefore, 0D nanoparticles are not suitable for flexible PDs. The distinct geometric structures of one-dimensional (1D) nanowires (NWs) and twodimensional (2D) nanosheets (NSs) make them reliable and suitable for flexible PDs [29-31]. For 1D NWs, they

Key Laboratory of Advanced Display Materials and Devices, Ministry of Industry and Information Technology, College of Material Science and Engineering, Nanjing University of Science and Technology, Nanjing 210094, China

* Corresponding authors (emails: yshzou75@njust.edu.cn (Zou Y); songjizhong@njust.edu.cn (Song J)) 
intertwine with each other to form a film of dense network that could resist external forces. Meanwhile, outstanding electrical properties of 1D NWs facilitate efficient carrier transport. The effective area of the carriers is limited and the transit time is shortened due to the reduced dimensionality, which enhances the photoresponse and optical gain [32-36]. Moreover, NW-based films can be easily transferred to flexible substrates. As a result, NW-based PDs have high performances and broad application prospects in the flexible fields. For example, Kind et al. [37] reported an ultraviolet PD based on an individual $\mathrm{ZnO} \mathrm{NW}$ and demonstrated its potential for optical switches. Wang et al. [38] described a $\mathrm{ZnGa}_{2} \mathrm{O}_{4}$ $\mathrm{PD}$ with a large photoresponse resulting from the Schottky barrier between the $\mathrm{Ag}$ and $\mathrm{ZnGa}_{2} \mathrm{O}_{4} \mathrm{NWs}$ and the junction barrier between interconnecting $\mathrm{ZnGa}_{2} \mathrm{O}_{4}$ NWs. Li et al. [39] demonstrated that PDs based on $\mathrm{ZnO}$ quantum dot-decorated $\mathrm{Zn}_{2} \mathrm{SnO}_{4}$ NWs exhibited the improved responsivity and outstanding flexibility. With respect to $2 \mathrm{D}$ materials, such as NSs, they would interconnect with each other in films, exhibiting superior flexibility and compatibility than quantum dots and bulk materials. Another advantage of $2 \mathrm{D}$ materials is that charges can be transported rapidly in plane due to smaller hopping barriers [40-41]. As a consequence, 2D materials display distinctive superiority in applications of flexible PDs. For example, Song et al. [42] reported 2D inorganic perovskite NSs for the first time and fabricated the PD with a high and fast photoresponse. Lim et al. [43] investigated a flexible PD based on a homogeneous $\mathrm{MoS}_{2}$ layer, which decreased only $5.6 \%$ in the photocurrent after bending for $10^{5}$ cycles. Thus, $2 \mathrm{D}$ NSs have also been proved as promising materials for flexible PDs.

With the rapid development of society and technology, it is difficult to satisfy the large demand for multifunctional detection by using a single material. Therefore, integrating different nanomaterials is a feasible strategy for constructing multifunctional devices. Herein, a highly flexible PD based on low-dimensional $\mathrm{ZnO} \mathrm{NWs}$ and $\mathrm{CsPbBr}{ }_{3}$ NSs is reported for the first time. The PD presents a low dark current, a high on/off ratio $\left(>10^{3}\right)$, good mechanical durability, and superior flexibility $(>1000$ cycles). Because $\mathrm{ZnO}$ is a typical semiconductor material with a bandgap of $3.3 \mathrm{eV}$ and absorption in the ultraviolet region, and $\mathrm{CsPbBr}_{3}$ is considered as an emerging material with excellent absorption in the visible region. Thus, the PD demonstrates broad photodetecting performance resulting from the integration of $\mathrm{ZnO}$ and $\mathrm{CsPbBr}_{3}$. The fabricated $\mathrm{PD}$ responses to light ranging from ultraviolet to visible region and presents high detectivities up to
$5.57 \times 10^{12}$ and $1.71 \times 10^{12}$ Jones at the wavelengths of 380 and $520 \mathrm{~nm}$, respectively.

\section{EXPERIMENTAL SECTION}

\section{Preparation of $\mathrm{CsPbBr}_{3}$ NSs}

The $\mathrm{CsPbBr}{ }_{3}$ NSs were synthesized via a solution process at room temperature. First, $0.1064 \mathrm{~g} \mathrm{CsBr}$ and $0.0918 \mathrm{~g}$ $\mathrm{PbBr}_{2}$ were dissolved in $7 \mathrm{~mL}$ dimethyl sulfoxide (DMSO) and $0.5 \mathrm{~mL}$ acetic acid as solution A. $1 \mathrm{~g}$ octadecylamine was dissolved in $5 \mathrm{~mL}$ acetic acid using an ultrasonic treatment for a few minutes as solution $\mathbf{B}$. Then $200 \mu \mathrm{L}$ of solution $\mathbf{A}$ and $1 \mathrm{~mL}$ of solution $\mathbf{B}$ were mixed in a $50-\mathrm{mL}$ centrifuge tube and stirred for $2 \mathrm{~min}$. Later, $20 \mathrm{~mL}$ toluene was added quickly into the tube. The mixture was stirred for another $3 \mathrm{~min}$ to form orange $\mathrm{CsPbBr}_{3} \mathrm{NSs}$. Finally, pure $\mathrm{CsPbBr}$ NSs were obtained by rinsing with toluene and centrifugation.

\section{Fabrication of the flexible PDs}

A polyethylene terephthalate (PET) substrate with $\mathrm{Au} / \mathrm{Cr}$ interdigital electrodes was used for constructing PDs. Firstly, $0.05 \mathrm{~g} \mathrm{ZnO}$ NWs (purchased from Hebei Shaojing New Material Technology Co., Ltd.) were dispersed in $20 \mathrm{~mL}$ isopropanol and $\mathrm{CsPbBr}_{3}$ NSs were dispersed in $4 \mathrm{~mL}$ toluene. Then $\mathrm{ZnO}$ NWs were deposited onto a polyvinylidenfluorid (PVDF) filtration membrane by suction filtration. Finally, the filtration membrane with the $\mathrm{ZnO}$ NWs and $\mathrm{CsPbBr}_{3}$ NSs was overturned and quickly transferred to the aforementioned PET/Au substrate by applying appropriate pressure. When the filtration membrane was uncovered, the $\mathrm{ZnO} / \mathrm{CsPbBr}_{3}$ film was laid onto the PET substrate.

\section{Characterization of materials and devices}

The morphologies of the NWs and NSs were observed by field emission scanning electron microscopy (FESEM, FEI Quanta 250F) and transmission electron microscopy (TEM, FEI Tecnai G20). The X-ray diffraction (XRD) patterns were obtained by using Bruker D8 Advance. The thickness of the $\mathrm{CsPbBr}_{3}$ NS was measured by a Bruker Multimode 8. The absorbance of the homogeneous dispersion was measured by using a Shimadzu $3600 \mathrm{UV}$-Vis spectrophotometer. The responsivity and external quantum efficiency (EQE) of PDs were measured through a Zolix DSR-101-UV detector responsivity measurement system. 320 and $532 \mathrm{~nm}$ continuous semiconductor lasers were used as light source to measure the optoelectronic properties of the PDs. A Keithley 6487 picoammeter was used to obtain the current. 


\section{RESULTS AND DISCUSSION}

\section{Morphologies and structures}

The crystal structures of the $\mathrm{ZnO}$ NWs and ultrathin $\mathrm{CsPbBr}_{3}$ NSs were analyzed by TEM and XRD. As shown in Fig. 1a and Fig. S1, the NW has a small diameter of approximately $100 \mathrm{~nm}$ and a large length to diameter ratio. The structural information was obtained by selected-area electron diffraction (SAED), as shown in the inset of Fig. 1a. The lattice spacing of $0.26 \mathrm{~nm}$ corresponds to the crystal plane (001) of $\mathrm{ZnO}$, and the $\mathrm{ZnO}$ NWs have a preferred growth orientation along the (002) direction. Fig. $1 \mathrm{~b}$ shows that the $2 \mathrm{D}$ quadrilateral $\mathrm{Cs} \mathrm{PbBr}_{3}$ NSs have clean and smooth surfaces. No other by-pro- ducts are observed, such as small-sized particles, which are common in the synthesis of $\mathrm{CsPbBr}_{3}$ nanocrystals [44]. The NSs have similar length and width of several hundred nanometers, and the thickness is approximately $8 \mathrm{~nm}$, as shown in the atomic force microscopy (AFM) image (Fig. S2). Furthermore, the NS in the bottom can be observed and the number of stacked layers of NSs can be determined. In addition, the wrinkles on the NSs are clear evidence of good flexibility. The insets in Fig. $1 \mathrm{~b}$ reveal that the lattice spacing is $0.58 \mathrm{~nm}$, corresponding to the (100) plane, which is in agreement with the XRD patterns presented in Fig. 1c. In the XRD patterns of the $\mathrm{ZnO} \mathrm{NWs}$ and the $\mathrm{ZnO} / \mathrm{CsPbBr}_{3}$ assembled film, no impurity peaks are observed, demonstrating superior
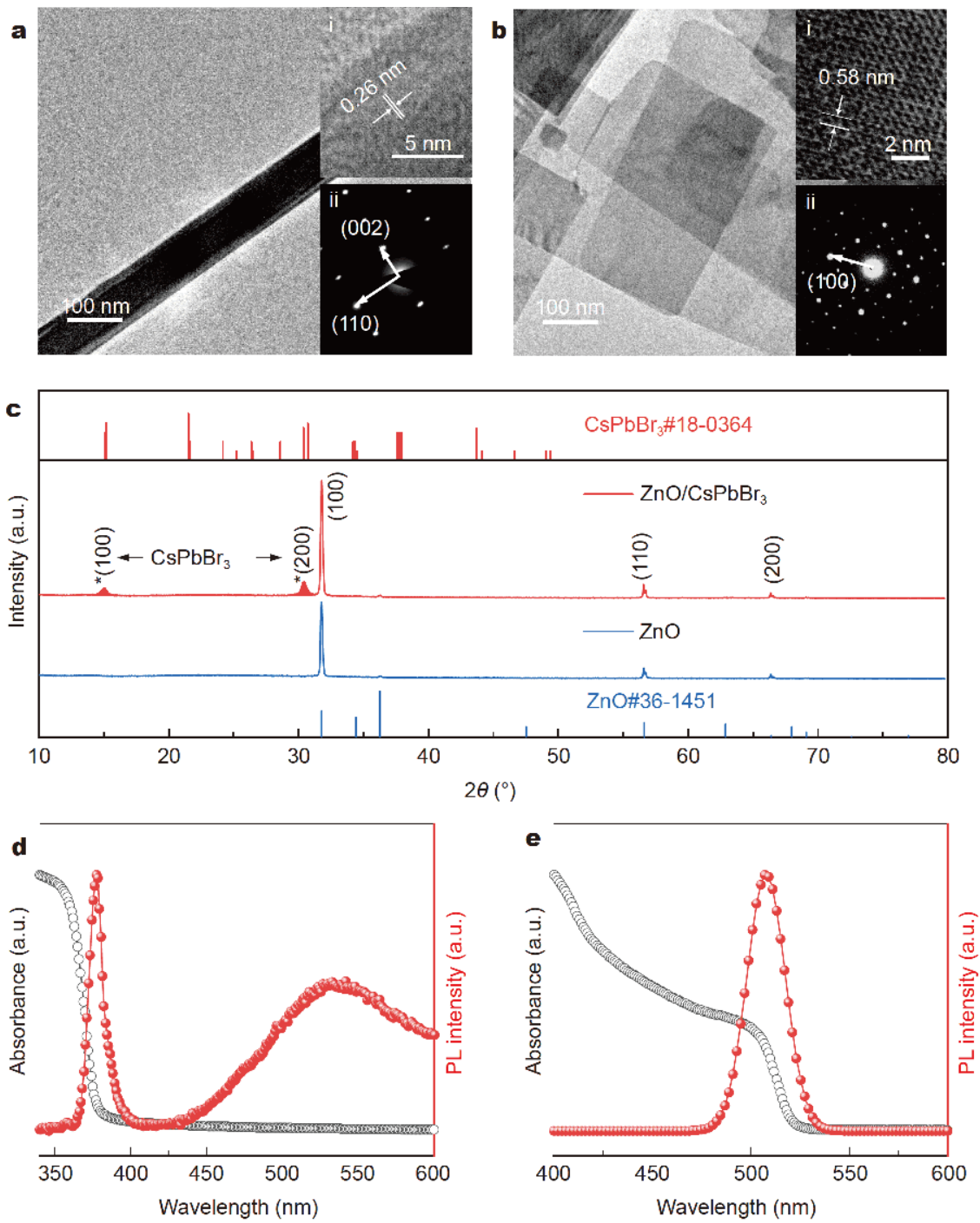

Figure 1 Typical TEM images of (a) ZnO NW and (b) $\mathrm{CsPbBr}_{3} \mathrm{NSs}$, insets are the corresponding (i) HRTEM and (ii) SAED. (c) XRD patterns of the pure $\mathrm{ZnO}$ NWs and $\mathrm{CsPBr}_{3} / \mathrm{ZnO}$ heterojunction film. Absorbance and PL spectra of (d) ZnO NWs and (e) $\mathrm{CsPbBr}_{3} \mathrm{NSs}_{\text {. }}$ 
purity of the $\mathrm{ZnO} \mathrm{NWs}$ and the $\mathrm{CsPbBr}_{3}$ NSs. The characteristic peaks of (100), (110), and (200) observed in both patterns are indexed to the wurtzite structure of $\mathrm{ZnO}$. A sharp (100) diffraction peak and two additional relatively small peaks indicate the typical orientation growth of $\mathrm{ZnO} \mathrm{NWs}$. Compared with the XRD pattern of the $\mathrm{ZnO} \mathrm{NWs}$, two additional peaks of (100) and (200) for $\mathrm{CsPbBr}_{3}$ (JSPDS \#18-0364) appeared. The XRD results indicate that the prepared $\mathrm{CsPbBr}_{3}$ NSs exhibit the typical layered structure, which is favorable for carrier transport in PDs.

\section{Optical properties}

The optical properties of the NWs and NSs were measured by using UV-Vis absorption and photoluminescence (PL) spectra. As seen in Fig. 1d, there is almost no absorption in the visible region, whereas the absorption sharply increases in the ultraviolet region. The exciton peak at $360 \mathrm{~nm}$ is attributed to the near-bandgap absorption of the $\mathrm{ZnO}$ NWs. The normalized PL spectrum of the $\mathrm{ZnO}$ NWs consists of two emission peaks, a near-band-edge (NBE) emission peak centered at $377 \mathrm{~nm}$ with a full width at half-maximum (FWHM) of $10 \mathrm{~nm}$, and a broad peak located in the visible region. Usually, the NBE emission is attributed to the collision between excitons, indicating the emergence and recombination of photo-generated carriers in the $\mathrm{ZnO}$ NWs. The wide peak around $530 \mathrm{~nm}$ is attributed to the deep-level emission from oxygen vacancies on the surface of the $\mathrm{ZnO}$ NWs [45]. The oxygen vacancies may absorb oxygen under the atmospheric environment [46-48], which improves the device performance to some extent. As shown in Fig. 1e, the prepared $\mathrm{Cs} \mathrm{PbBr}_{3}$ NSs exhibit an absorbance edge at approximately $520 \mathrm{~nm}$, which is consistent with the previous results $[49,50]$. The corresponding PL spectrum shows a highly symmetric emission peak at $520 \mathrm{~nm}$. Moreover, the emission peak has a narrow linewidth of $22 \mathrm{~nm}$ and no additional sub-bandgap emission is observed, indicating the high quality of the $\mathrm{CsPbBr}_{3} \mathrm{NSs}$, which is favorable for constructing high-performance devices.

\section{Device construction}

The flexible PD was constructed by combining $\mathrm{ZnO}$ NWs and $\mathrm{CsPbBr}_{3}$ NSs (Fig. 2). First, the $\mathrm{ZnO}$ NWs were deposited on the PVDF filter membrane by a vacuum-filtration route. The corresponding SEM image shows that the highly dense NWs possess a very long length beyond tens of microns and are naturally intertwined with each other. Next, the CsPbBr ${ }_{3}$ NSs were deposited on the existing $\mathrm{ZnO} \mathrm{NWs}$ by vacuum-filtration route. As shown in the SEM image, the NSs deposited on the ZnO NWs are spread out and interlinked. Finally, the assembled film combined with the $\mathrm{ZnO} \mathrm{NWs}$ and the $\mathrm{CsPbBr}_{3}$ NSs was transferred onto the PET substrate with $\mathrm{Au} / \mathrm{Cr}$ interdigital electrodes. The SEM image of the fabricated device demonstrates the morphology of the device with copious $\mathrm{ZnO}$ NWs. The Au electrodes can be clearly observed. However, the $\mathrm{CsPbBr}_{3}$ NSs at the bottom are not clear due to the overlapping $\mathrm{ZnO}$ NWs. This transfer method can be used for assembling compact film on various substrates.

\section{The flexibility of the device}

The flexibility and bendability of the fabricated PD were then evaluated. Fig. 3a presents a typical photograph of the flexible $\mathrm{ZnO} / \mathrm{CsPbBr}_{3} \mathrm{PD}$. To assess the reliability of the fabricated devices, we compared the device performances before and after repeated mechanical bending. The SEM images of the device before and after bending 1000 times are shown in Fig. 3b and c, where no apparent change is observed, indicating the excellent bendability of the PDs. In addition, the photocurrents under periodical illumination (Fig. 3d) also exhibit no significant at-

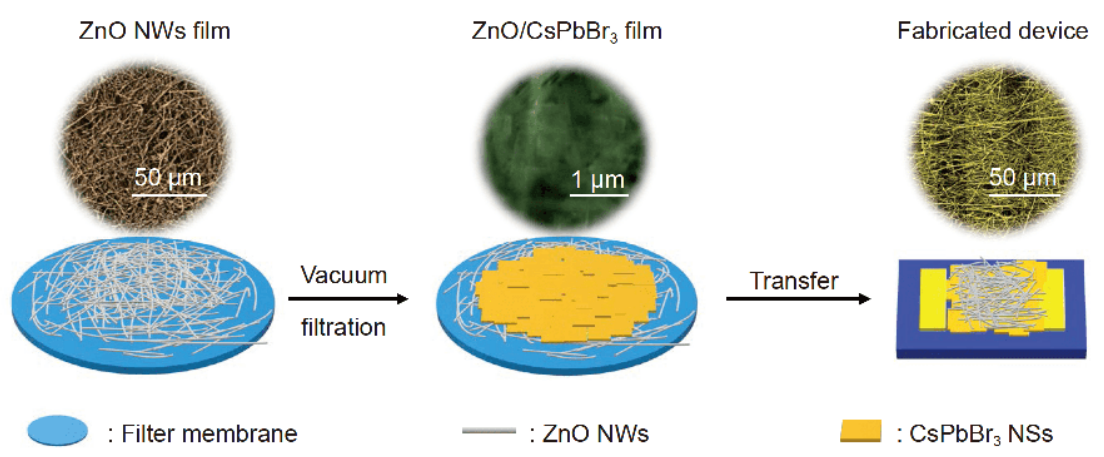

Figure 2 Schematic diagrams of the fabrication process of the PD and the corresponding morphologies of the sample at each step. 

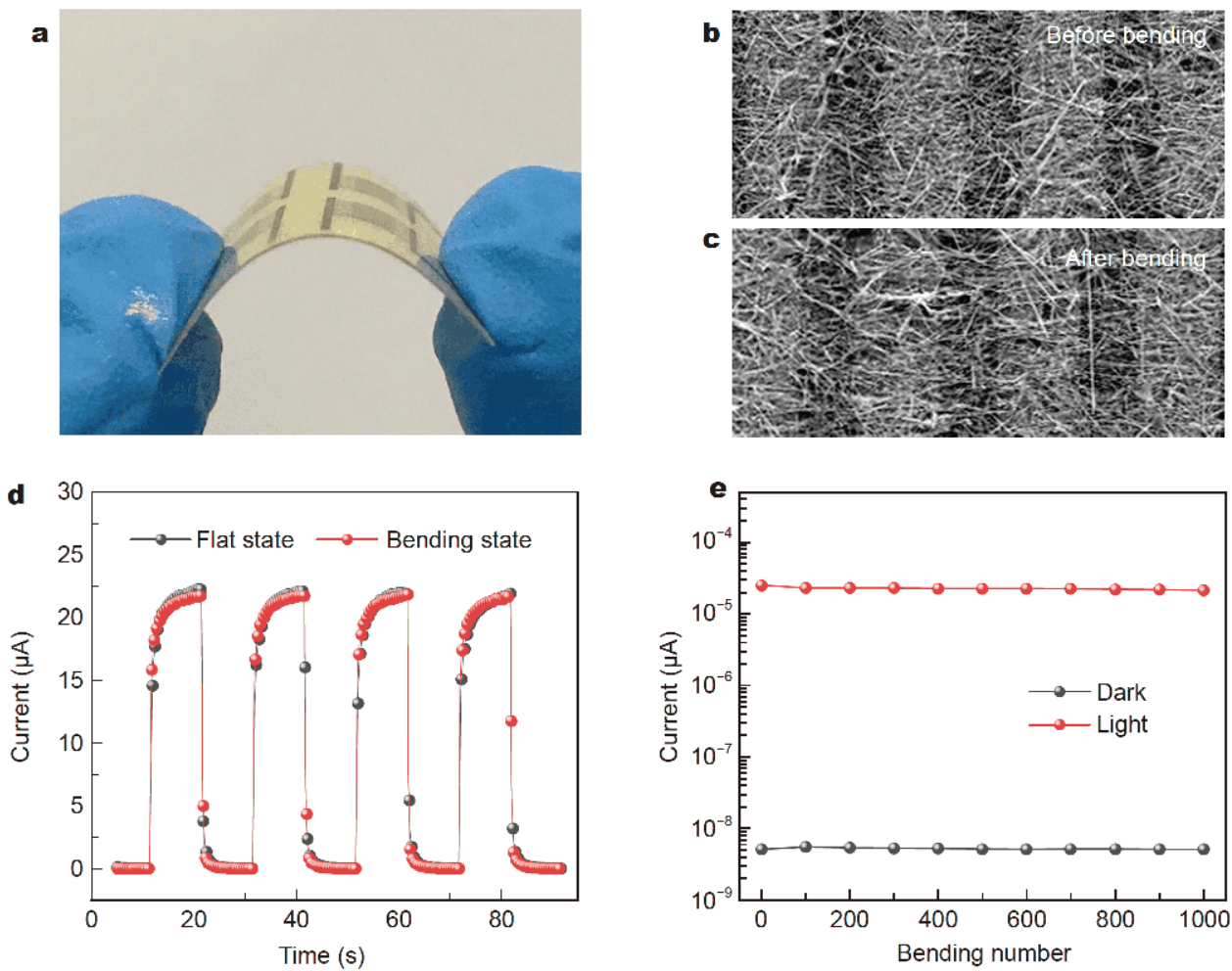

Figure 3 The bending performance of the flexible PDs. (a) The photograph of the flexible PD. SEM images of the flexible PD (b) before and (c) after bending. (d) $I-t$ curves at $1 \mathrm{~V}$ of the flexible PD under flat and bending state. (e) The photoresponse of the flexible PD under different bending times.

tenuation in the bent state compared to the flat state, demonstrating excellent stability and reversibility. The slight change in the photocurrent may be the result of the contacts between the electrodes and probes when the device changes from the flat state to the bent state. Moreover, the photocurrent of the flexible PD under $1 \mathrm{~mW}$ illumination after bending for 1000 times (Fig. 3e) is almost the same as that of the initial device. This further demonstrates that the device is adaptable to the bending stress. The behavior of the flexible PD at different bending degrees was also evaluated (Fig. S3). The photographs in the inset show the bending degree of the device. At different bending degrees, the dark currents show no change while the photocurrents show a slight fluctuation. These observations indicate that the fabricated flexible PD based on the $\mathrm{ZnO} / \mathrm{CsPbBr}_{3}$ heterojunction is able to bear an external mechanical force, which can be attributed to the flexibility of the unique low-dimensional nanostructures.

\section{Broadband response of the device}

Due to the advantages of the integration of $\mathrm{ZnO}$ and $\mathrm{CsPbBr}_{3}$, the PD can potentially exhibit a broad photo- response. Fig. 4a shows the absorption of the $\mathrm{ZnO} /$ $\mathrm{Cs} \mathrm{PbBr}_{3}$ composite, which has a wider range than the $\mathrm{ZnO}$ NWs including the visible region due to the introduction of the $\mathrm{CsPbBr}_{3}$ NSs. Compared with $\mathrm{ZnO}$ NWs and $\mathrm{CsPbBr}_{3}$ NSs, the PL spectrum of $\mathrm{ZnO} / \mathrm{CsPbBr}_{3}$ (Fig. S4a) exhibits no obvious shift. However, we find that the PL lifetime of the $\mathrm{ZnO} / \mathrm{CsPbBr}_{3}$ is shorter than that of pure $\mathrm{Cs} \mathrm{PbBr}_{3}$, which is attributed to the transfer of the carriers from $\mathrm{CsPbBr}_{3}$ to $\mathrm{ZnO}$. Thus, the carriers can be easily separated and transported to the electrode, which would improve the photoelectric properties of the flexible $\mathrm{PD}$ (Fig. S4b). The unique optical properties of the $\mathrm{ZnO} /$ $\mathrm{CsPbBr}$-assembled film make it an ideal candidate for broad-spectrum PDs.

The responsivity $(R)$ is one of the most crucial parameters for evaluating the photoresponse of a PD. $R$ is defined in the following equation [51],

$R=\frac{I_{\text {photo }}}{P_{\text {light }} \times A}$,

where $I_{\text {photo }}, P_{\text {light }}$ and $A$ are the photocurrent, irradiation power density and working area of the $\mathrm{PD}$, respectively. The responsivities were measured under different bias 

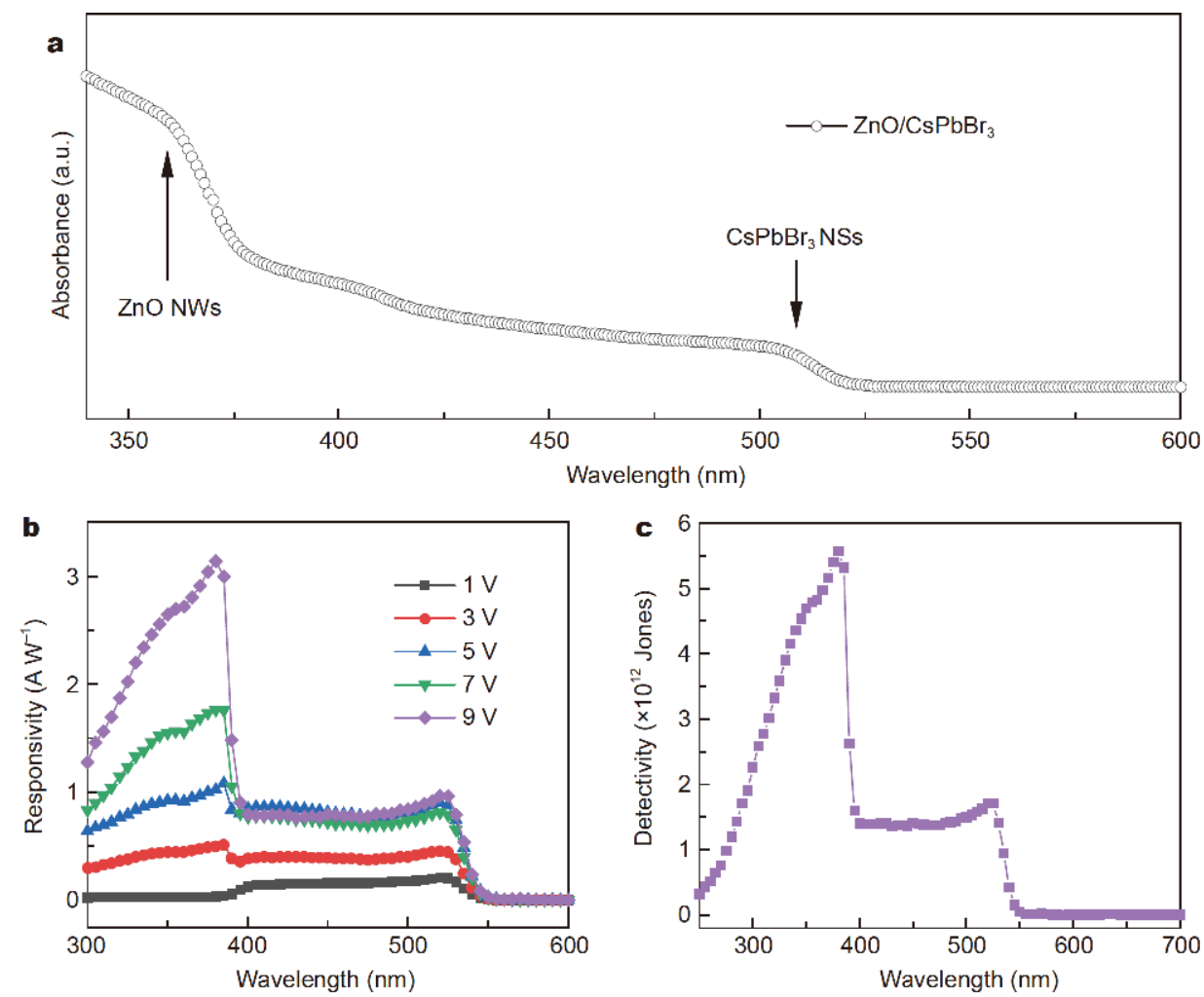

Figure 4 The broad spectral characteristics of the flexible PD. (a) Absorbance of the $\mathrm{ZnO} / \mathrm{CsPbBr}{ }_{3}$ composite. (b) Responsivity spectrum of $\mathrm{ZnO} /$ $\mathrm{CsPBr}_{3}$ heterojunction PD under different applied bias. (c) Detectivity of the PD under $9 \mathrm{~V}$.

voltages using a standard Si PD as a reference, as shown in Fig. 4b. The broad-responsivity spectra of the flexible $\mathrm{PD}$ cover a wavelength range from $300 \mathrm{~nm}$ (ultraviolet region) to $550 \mathrm{~nm}$ (visible region), which is consistent with the UV-Vis absorption spectrum. Normally, the peak located at $380 \mathrm{~nm}$ is attributed to the $\mathrm{ZnO} \mathrm{NWs}$ (shown in Fig. S5a) and the $520 \mathrm{~nm}$ peak is attributed to the $\mathrm{CsPbBr}_{3}$ NSs (shown in Fig. S5b). The sharp increases below 380 and $520 \mathrm{~nm}$ are owing to the different concentrations of electron-hole pairs excited by various wavelengths of light. The photoconduction mechanism of the PD is based on the production of photo-generated carriers (electron-hole pairs), which is motivated by incident light with greater photon energy than the bandgap. Meanwhile, the responsivity increases with increasing bias voltage, reaching $3.1 \mathrm{~A} \mathrm{~W}^{-1}$ at $380 \mathrm{~nm}$ and $0.97 \mathrm{~A} \mathrm{~W}^{-1}$ at $520 \mathrm{~nm}$ under $9 \mathrm{~V}$, which are higher than those of the reference Si PD $\left(<0.2 \mathrm{~A} \mathrm{~W}^{-1}\right)$. The EQE (shown in Fig. S6) exhibits a high numerical value of $1024 \%$ at $380 \mathrm{~nm}$ and $230 \%$ at $520 \mathrm{~nm}$ under $9 \mathrm{~V}$. The high EQE value is attributed to a large number of photogenerated carriers under irradiation and the additional charges from the applied voltage.
Assuming that the short noise from the dark current acts as the major noise, the detectivity $\left(D^{*}\right)[52]$ is calculated as:

$D^{*}=\frac{R \times A^{1 / 2}}{\left(2 \mathrm{e} \cdot I_{\text {dark }}\right)^{1 / 2}}$

where e refers to the electron charge. The relationship between the calculated detectivity and the wavelength is shown in Fig. 4c. The detectivities at 380 and $520 \mathrm{~nm}$ are $5.57 \times 10^{12}$ and $1.71 \times 10^{12}$ Jones, respectively. This is comparable to the reported state-of-the-art PDs with broadband photoresponse [53-56].

\section{Mechanism of charge transport}

The current-voltage $(I-V)$ characteristics of the $\mathrm{ZnO} /$ $\mathrm{CsPbBr}$ heterojunction PD were measured in dark and light conditions (the power density of the applied laser was $1 \mathrm{~mW} \mathrm{~cm}^{-2}$ ), respectively. As shown in Fig. 5a, the photocurrents under illumination exhibit a large increase compared with the dark current and an excellent photoresponse is observed in the UV-vis region. The extracted dark current from the $I-V$ logarithmic curve (Fig. 5b) is approximately $5.9 \times 10^{-10} \mathrm{~A}$ under an applied 

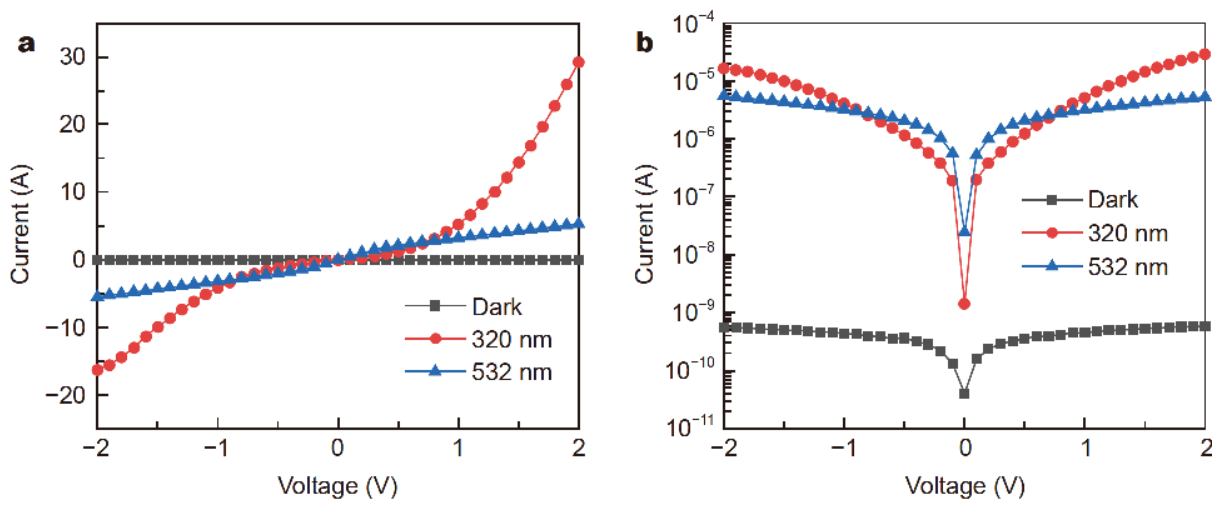

Figure 5 (a) $I-V$ characteristics and (b) $I-V$ logarithmic characteristics of the flexible $\mathrm{ZnO} / \mathrm{CsPbBr}{ }_{3}$ heterojunction $\mathrm{PD}$ under dark and illumination.

voltage of $2 \mathrm{~V}$, while the photocurrents under irradiation of 320 and $532 \mathrm{~nm}$ are $2.9 \times 10^{-5}$ and $5.2 \times 10^{-6} \mathrm{~A}$, respectively. The on/off ratios, which are defined as $\left(I_{\text {photo }}-I_{\text {dark }}\right) /$ $I_{\text {dark }}$, are $4.91 \times 10^{4}$ and $8.81 \times 10^{3}$, respectively. These high on/off ratios are the result of the high light absorbance of the $\mathrm{ZnO} \mathrm{NWs}$ in the ultraviolet region and the $\mathrm{CsPbBr}_{3}$ NSs in the visible region. The perovskite/ZnO heterojunction PDs have been reported and some of them have demonstrated excellent responsivity, detectivity, and high on/off ratios, as listed in Table 1. These high-performance $\mathrm{PDs}$ based on perovskite/ZnO were usually realized on rigid substrates. However, flexible PDs with high optoelectronic properties are relatively scarce on account of substrate compatibility and mechanical stability. In this study, both excellent flexibility and high performance are achieved by using low-dimensional structures.

In order to illustrate the underlying mechanism of the broad photoresponse of the flexible PD, schematic diagrams of the carrier transport are presented in Fig. 6. The band structures of the PD are shown in Fig. 6a and c, demonstrating that a staggered heterojunction of $\mathrm{ZnO} /$ $\mathrm{CsPbBr}{ }_{3}$ is formed. Fig. $6 \mathrm{~b}$ and $\mathrm{d}$ show the transport paths of the electron and hole in the PD under illumination. Under illumination of $320 \mathrm{~nm}$, photo-generated carriers are mainly generated in the $\mathrm{ZnO}$ NWs. Because most of the $\mathrm{CsPbBr}$ NSs are covered by the $\mathrm{ZnO}$ NWs and hence the light absorbance mainly occurs in the $\mathrm{ZnO}$ NWs. Relatively, the valence band of the $\mathrm{ZnO}$ lies lower than that of the $\mathrm{CsPBr}_{3}$, the photo-generated holes tend to migrate to the $\mathrm{CsPBr}_{3}$ NSs, resulting in efficient separation of the photo-generated carriers. Then, carriers pass through the NSs, and are collected by the Au electrode. Under illumination of $532 \mathrm{~nm}$, the long wavelength light normally has a deeper penetration ability. The light can pass through the ZnO NWs and reach the NSs. Because the photon energy is much lower than the bandgap of $\mathrm{ZnO}$, which cannot produce photo-generated carriers in $\mathrm{ZnO}$. Thus, the photo-generated carriers are mainly generated in the $\mathrm{CsPbBr}_{3}$ NSs and are collected by the electrodes. The different photo-generated carrier features of $\mathrm{ZnO} \mathrm{NW/CsPbBr}{ }_{3} \mathrm{NS}$ structure make the flexible PD with a broad photoresponse.

\section{CONCLUSION}

In summary, a mechanically stable and flexible PD based on $\mathrm{ZnO} \mathrm{NWs} / \mathrm{CsPbBr}{ }_{3}$ NSs was designed by using a simple vacuum-filtration route. The optoelectronic properties of PD exhibited almost no change after bending for 1000 times. Importantly, as a result of the different light absorbance characteristics of $\mathrm{ZnO}$ and

Table 1 Performance comparisons of perovskite/ $\mathrm{ZnO}$ nanostructure PDs

\begin{tabular}{ccccccc}
\hline Materials & Substrates & Wavelength $(\mathrm{nm})$ & Responsivity $\left(\mathrm{A} \mathrm{W}^{-1}\right)$ & Detectivity (Jones) & On/off ratio & Ref. \\
\hline $\mathrm{CH}_{3} \mathrm{NH}_{3} \mathrm{PbI}_{3} / \mathrm{ZnO}$ nanorod array & FTO glass & 500 & 24.3 & $3.56 \times 10^{12}$ & - & {$[57]$} \\
$\mathrm{CsPbBr} / \mathrm{ZnO}$ & PET & 450 & 4.25 & & $>10^{4}$ & {$[58]$} \\
$\mathrm{ZnO}$ nanofiber/perovskite & ITO glass & & 0.1 & $10^{13}$ & $2.80 \times 10^{3}$ & {$[59]$} \\
$\mathrm{CH}_{3} \mathrm{NH}_{3} \mathrm{PbI}_{3} / \mathrm{ZnO}$ & Mica & 380 & 4 & $1.74 \times 10^{9}$ & 8 & {$[60]$} \\
$\mathrm{CsPBr}_{3} \mathrm{NSs} / \mathrm{ZnO} \mathrm{NWs}$ & PET & 380 & 3.1 & $5.57 \times 10^{12}$ & $4.91 \times 10^{4}$ & This work \\
\hline
\end{tabular}



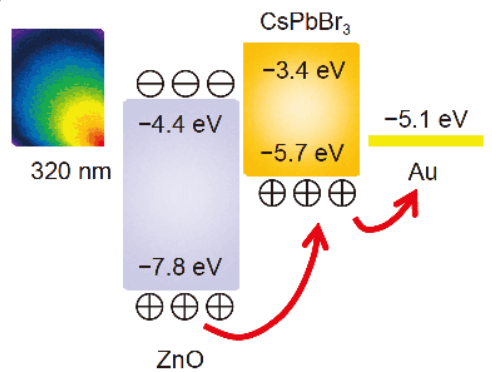

C

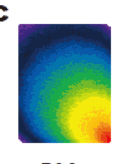

$532 \mathrm{~nm}$

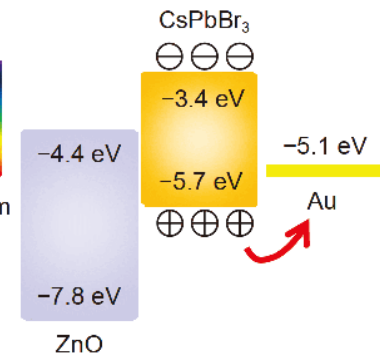

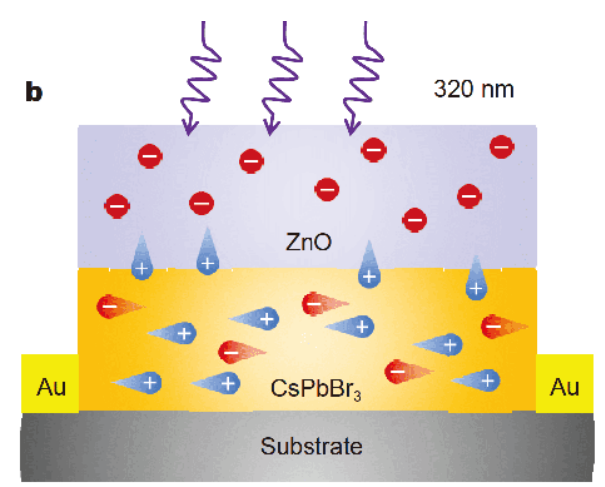

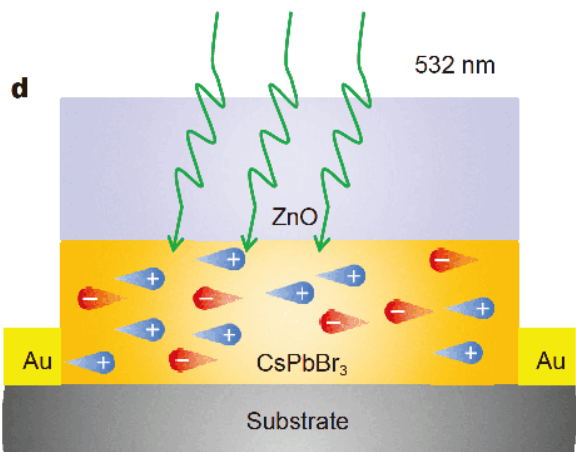

Figure 6 The energy band diagram in $\mathrm{ZnO} / \mathrm{CsPbBr}_{3}$ heterojunction under illumination of (a) $320 \mathrm{~nm}$ and (c) $532 \mathrm{~nm}$, respectively. The schematic diagram of the carrier transport in $\mathrm{ZnO} / \mathrm{CsPbBr}_{3}$ heterojunction under illumination of (b) $320 \mathrm{~nm}$ and (d) $532 \mathrm{~nm}$, respectively.

$\mathrm{CsPbBr}_{3}$, the flexible PD exhibited a broadband photoresponse. High responsivities of 3.10 and $0.97 \mathrm{~A} \mathrm{~W}^{-1}$ and high detectivities of $5.57 \times 10^{12}$ and $1.71 \times 10^{12}$ Jones were obtained in the ultraviolet and visible regions, respectively. These results demonstrate that the low-dimensional materials show great advantages in flexible optoelectronic devices, which makes them a favorable candidate in the fields of wearable electronic devices, folding displays and other applications.

\section{Received 1 April 2019; accepted 16 May 2019;} published online 12 June 2019

1 Hu PA, Wang L, Yoon M, et al. Highly responsive ultrathin gas nanosheet photodetectors on rigid and flexible substrates. Nano Lett, 2013, 13: 1649-1654

2 Tian W, Zhang C, Zhai T, et al. Flexible ultraviolet photodetectors with broad photoresponse based on branched $\mathrm{ZnS}-\mathrm{ZnO}$ heterostructure nanofilms. Adv Mater, 2014, 26: 3088-3093

3 Zhou X, Zhang Q, Gan L, et al. Large-Size growth of ultrathin $\mathrm{SnS}_{2}$ nanosheets and high performance for phototransistors. Adv Funct Mater, 2016, 26: 4405-4413

4 Wang S, Zou Y, Shan Q, et al. Nanowire network-based photodetectors with imaging performance for omnidirectional photodetecting through a wire-shaped structure. RSC Adv, 2018, 8: 33666-33673

5 Lou Z, Li L, Shen G. High-performance rigid and flexible ultra- violet photodetectors with single-crystalline $\mathrm{ZnGa}_{2} \mathrm{O}_{4}$ nanowires. Nano Res, 2015, 8: 2162-2169

6 Xie C, Yan F. Flexible photodetectors based on novel functional materials. Small, 2017, 13: 1701822

7 Wang X, Song W, Liu B, et al. High-performance organicinorganic hybrid photodetectors based on P3HT:CdSe nanowire heterojunctions on rigid and flexible substrates. Adv Funct Mater, 2013, 23: 1202-1209

8 Choi S, Lee H, Ghaffari R, et al. Recent advances in flexible and stretchable bio-electronic devices integrated with nanomaterials. Adv Mater, 2016, 28: 4203-4218

9 Xue J, Chen J, Song J, et al. Wearable and visual pressure sensors based on $\mathrm{Zn}_{2} \mathrm{GeO}_{4} @$ polypyrrole nanowire aerogels. J Mater Chem C, 2017, 5: 11018-11024

10 Gao W, Emaminejad S, Nyein HYY, et al. Fully integrated wearable sensor arrays for multiplexed in situ perspiration analysis. Nature, 2016, 529: 509-514

11 Amjadi M, Pichitpajongkit A, Lee S, et al. Highly stretchable and sensitive strain sensor based on silver nanowire-elastomer nanocomposite. ACS Nano, 2014, 8: 5154-5163

12 Shin SS, Yang WS, Noh JH, et al. High-performance flexible perovskite solar cells exploiting $\mathrm{Zn}_{2} \mathrm{SnO}_{4}$ prepared in solution below $100^{\circ} \mathrm{C}$. Nat Commun, 2015, 6: 7410

13 Kaltenbrunner M, Adam G, Głowacki ED, et al. Flexible high power-per-weight perovskite solar cells with chromium oxidemetal contacts for improved stability in air. Nat Mater, 2015, 14: 1032-1039

14 Wang Y, Bai S, Cheng L, et al. High-efficiency flexible solar cells based on organometal halide perovskites. Adv Mater, 2016, 28: 
4532-4540

15 Kim T, Kim JH, Kang TE, et al. Flexible, highly efficient allpolymer solar cells. Nat Commun, 2015, 6: 8547

16 Zang Y, Zhang F, Huang D, et al. Flexible suspended gate organic thin-film transistors for ultra-sensitive pressure detection. Nat Commun, 2015, 6: 6269

17 Yang Y, Yang X, Zou X, et al. Ultrafine graphene nanomesh with large on/off ratio for high-performance flexible biosensors. Adv Funct Mater, 2017, 27: 1604096

18 . Xiang L, Wang Z, Liu Z, et al. Inkjet printed flexible biosensor based on graphene field effect transistor. IEEE Senss J, 2016, 16: 8359

19 Petrone N, Chari T, Meric I, et al. Flexible graphene field-effect transistors encapsulated in hexagonal boron nitride. ACS Nano, 2015, 9: 8953-8959

20 Lee SH, Kim SB, Moon YJ, et al. High-responsivity deep-ultraviolet-selective photodetectors using ultrathin gallium oxide films. ACS Photonics, 2017, 4: 2937-2943

21 Jayasinghe RC, Ariyawansa G, Dietz N, et al. Simultaneous detection of ultraviolet and infrared radiation in a single GaN/GaAlN heterojunction. Opt Lett, 2008, 33: 2422-2424

22 Monroy E, Omn s F, Calle F. Wide-bandgap semiconductor ultraviolet photodetectors. Semicond Sci Technol, 2003, 18: R33-R51

23 Liu K, Sakurai M, Aono M. ZnO-based ultraviolet photodetectors. Sensors, 2010, 10: 8604-8634

24 Su L, Yang W, Cai J, et al. Self-powered ultraviolet photodetectors driven by built-in electric field. Small, 2017, 13: 1701687

25 Dong Y, Wang S, Zou Y, et al. Zinc stannate nanocrystal-based ultrarapid-response UV photodetectors. Adv Mater Technol, 2018, 3: 1800085

26 Cheng W, Niederberger M. Evaporation-induced self-assembly of ultrathin tungsten oxide nanowires over a large scale for ultraviolet photodetector. Langmuir, 2016, 32: 2474-2481

27 Li X, Yu D, Cao F, et al. Healing all-inorganic perovskite films via recyclable dissolution-recyrstallization for compact and smooth carrier channels of optoelectronic devices with high stability. Adv Funct Mater, 2016, 26: 5903-5912

28 Sang L, Liao M, Sumiya M. A comprehensive review of semiconductor ultraviolet photodetectors: From thin film to onedimensional nanostructures. Sensors, 2013, 13: 10482-10518

29 Liu Z, Xu J, Chen D, et al. Flexible electronics based on inorganic nanowires. Chem Soc Rev, 2015, 44: 161-192

30 Yan R, Gargas D, Yang P. Nanowire photonics. Nat Photon, 2009, 3: 569-576

31 Li Y, Qian F, Xiang J, et al. Nanowire electronic and optoelectronic devices. Mater Today, 2006, 9: 18-27

32 Peng $M$, Wen $Z$, Shao $M$, et al. One-dimensional $\mathrm{CdS}_{x} \mathrm{Se}_{1-x}$ nanoribbons for high-performance rigid and flexible photodetectors. J Mater Chem C, 2017, 5: 7521-7526

33 Shahid M, Cheng J, Li T, et al. High photodetectivity of lowvoltage flexible photodetectors assembled with hybrid aligned nanowire arrays. J Mater Chem C, 2018, 6: 6510-6519

34 Um DS, Lee $\mathrm{Y}$, Lim S, et al. High-performance $\mathrm{MoS}_{2} / \mathrm{CuO}$ nanosheet-on-one-dimensional heterojunction photodetectors. ACS Appl Mater Interfaces, 2016, 8: 33955-33962

35 Liang FX, Wang JZ, Li ZP, et al. Near-infrared-light photodetectors based on one-dimensional inorganic semiconductor nanostructures. Adv Opt Mater, 2017, 5: 1700081

36 Yang $\mathrm{X}$, Liu $\mathrm{Y}$, Lei $\mathrm{H}$, et al. An organic-inorganic broadband photodetector based on a single polyaniline nanowire doped with quantum dots. Nanoscale, 2016, 8: 15529-15537

37 Kind $\mathrm{H}$, Yan H, Messer B, et al. Nanowire ultraviolet photodetectors and optical switches. Adv Mater, 2002, 14: 158-160

38 Wang J, Yan C, Lin MF, et al. Solution-assembled nanowires for high performance flexible and transparent solar-blind photodetectors. J Mater Chem C, 2015, 3: 596-600

$39 \mathrm{Li} \mathrm{L}, \mathrm{Gu} \mathrm{L}$, Lou $\mathrm{Z}$, et al. $\mathrm{ZnO}$ quantum dot decorated $\mathrm{Zn}_{2} \mathrm{SnO}_{4}$ nanowire heterojunction photodetectors with drastic performance enhancement and flexible ultraviolet image sensors. ACS Nano, 2017, 11: 4067-4076

40 Lv L, Xu Y, Fang H, et al. Generalized colloidal synthesis of highquality, two-dimensional cesium lead halide perovskite nanosheets and their applications in photodetectors. Nanoscale, 2016, 8: 13589-13596

41 Li H, Zhitomirsky D, Grossman JC. Tunable and energetically robust $\mathrm{PbS}$ nanoplatelets for optoelectronic applications. Chem Mater, 2016, 28: 1888-1896

42 Song J, Xu L, Li J, et al. Monolayer and few-layer all-inorganic perovskites as a new family of two-dimensional semiconductors for printable optoelectronic devices. Adv Mater, 2016, 28: 4861-4869

43 Lim YR, Song W, Han JK, et al. Wafer-scale, homogeneous $\mathrm{MoS}_{2}$ layers on plastic substrates for flexible visible-light photodetectors. Adv Mater, 2016, 28: 5025-5030

44 Dong Y, Gu Y, Zou Y, et al. Improving all-inorganic perovskite photodetectors by preferred orientation and plasmonic effect. Small, 2016, 12: 5622-5632

45 Dong Y, Zou Y, Song J, et al. An all-inkjet-printed flexible UV photodetector. Nanoscale, 2017, 9: 8580-8585

46 Vanheusden $\mathrm{K}$, Warren WL, Seager $\mathrm{CH}$, et al. Mechanisms behind green photoluminescence in $\mathrm{ZnO}$ phosphor powders. J Appl Phys, 1996, 79: 7983-7990

47 Lin B, Fu Z, Jia Y. Green luminescent center in undoped zinc oxide films deposited on silicon substrates. Appl Phys Lett, 2001, 79: 943-945

48 Kennedy J, Murmu PP, Manikandan E, et al. Investigation of structural and photoluminescence properties of gas and metal ions doped zinc oxide single crystals. J Alloys Compd, 2014, 616: 614617

49 Yu D, Cai B, Cao F, et al. Cation exchange-induced dimensionality construction: From monolayered to multilayered 2D single crystal halide perovskites. Adv Mater Interfaces, 2017, 4: 1700441

50 Liu X, Yu D, Cao F, et al. Low-voltage photodetectors with high responsivity based on solution-processed micrometer-scale allinorganic perovskite nanoplatelets. Small, 2017, 13: 1700364

51 Peng M, Wang Y, Shen Q, et al. High-performance flexible and broadband photodetectors based on $\mathrm{PbS}$ quantum dots/ $\mathrm{ZnO}$ nanoparticles heterostructure. Sci China Mater, 2019, 62: 225-235

52 Peng $\mathrm{M}$, Xie $\mathrm{X}$, Zheng $\mathrm{H}$, et al. $\mathrm{PbS}$ quantum dots/2D nonlayered $\mathrm{CdS}_{x} \mathrm{Se}_{1-x}$ nanosheet hybrid nanostructure for high-performance broadband photodetectors. ACS Appl Mater Interfaces, 2018, 10: 43887-43895

53 Alwadai N, Haque MA, Mitra S, et al. High-performance ultraviolet-to-infrared broadband perovskite photodetectors achieved via inter-/intraband transitions. ACS Appl Mater Interfaces, 2017, 9: 37832-37838

54 Sun H, Tian W, Cao F, et al. Ultrahigh-performance self-powered flexible double-twisted fibrous broadband perovskite photodetector. Adv Mater, 2018, 30: 1706986

55 Sahatiya P, Reddy K CS, Badhulika S. Discretely distributed 1D $\mathrm{V}_{2} \mathrm{O}_{5}$ nanowires over 2D $\mathrm{MoS}_{2}$ nanoflakes for an enhanced 
broadband flexible photodetector covering the ultraviolet to near infrared region. J Mater Chem C, 2017, 5: 12728-12736

56 Wang P, Liu S, Luo W, et al. Arrayed van der Waals broadband detectors for dual-band detection. Adv Mater, 2017, 29: 1604439

57 Yu J, Chen X, Wang Y, et al. A high-performance self-powered broadband photodetector based on a $\mathrm{CH}_{3} \mathrm{NH}_{3} \mathrm{PbI}_{3}$ perovskite/ZnO nanorod array heterostructure. J Mater Chem C, 2016, 4: 73027308

58 Liu H, Zhang X, Zhang L, et al. A high-performance photodetector based on an inorganic perovskite- $\mathrm{ZnO}$ heterostructure. J Mater Chem C, 2017, 5: 6115-6122

59 Cao F, Tian W, Gu B, et al. High-performance UV-vis photodetectors based on electrospun $\mathrm{ZnO}$ nanofiber-solution processed perovskite hybrid structures. Nano Res, 2017, 10: 2244-2256

60 Gao T, Zhang Q, Chen J, et al. Performance-enhancing broadband and flexible photodetectors based on perovskite/ZnO-nanowire hybrid structures. Adv Opt Mater, 2017, 5: 1700206

Acknowledgements This work was financially supported by the National Natural Science Foundation of China (51672132 and 61604074), the Natural Science Foundation of Jiangsu Province (BK20160827 and BK20180020), China Postdoctoral Science Foundation (2016M590455), Open foundation of Key Laboratory of Marine Materials and Related Technologies (2016K08), the Fundamental Research Funds for the Central Universities (30917011202), and PAPD of Jiangsu Higher Education Institutions.

Author contributions Song J supervised the project; Wang S performed the experiments and wrote the manuscript; Zhu Z and Liu S took part in the fabrication and the characterization of $\mathrm{ZnO}$ NWs film; Zou Y and Song J performed the data analysis and revised the manuscript; Dong Y took part in the characterizations of the samples; Xue J and $\mathrm{Xu} \mathrm{L}$ took part in the preparation of $\mathrm{CsPbBr}_{3} \mathrm{NSs}$; Dong Y tested the $I-V$ and $I-t$ plots of the flexible photodetectors. All authors contributed to the general discussion.

Conflict of interest The authors declare no conflict of interest.

Supplementary information Supplementary results are available in the online version of the paper.

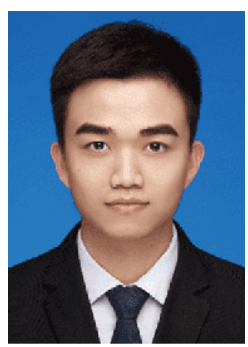

Shalong Wang received his BE from the School of Materials Science and Engineering, Nanjing University of Science and Technology, China. He is currently a $\mathrm{PhD}$ candidate at Nanjing University of Science and Technology. His current research focuses on nano-optoelectronic devices.

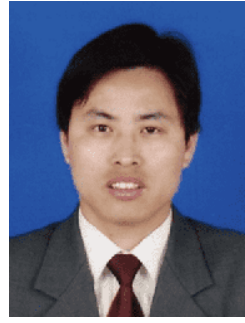

Yousheng Zou is a professor at the School of Materials Science and Engineering, Nanjing University of Science and Technology, China. He received his Doctoral degree from the Institute of Metal Research, Chinese Academy of Sciences, China. His current research interests are in the areas of optoelectronic films and devices, semiconductor nanocrystals, diamond films and applications.

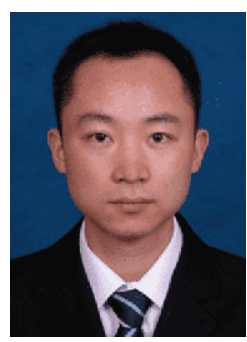

Jizhong Song received his Master degree in material science and engineering from Shanghai University in 2011, and $\mathrm{PhD}$ in material science from Nanjing University of Aeronautics and Astronautics in 2015. He is currently a professor at Nanjing University of Science and Technology. His current research focuses on colloidal nanocrystals, all inorganic halide perovskites and their optoelectronic applications in light-emitting diodes and photodetectors.

\title{
低维结构策略构筑基于钻钠矿纳米片/氧化锌纳 米线的柔性宽光谱探测器
}

\author{
王沙龙, 朱正峰, 邹友生”, 董宇航, 刘舒婷, 薛洁, 许雷梦, \\ 董宇辉, 宋继中
}

摘要 柔性光电探测器具有轻便、易携带和优异的大面积兼容性 等特点, 在下一代光电子器件领域具有巨大的应用潜力. 柔性光电 探测器面临的主要挑战是在反复弯曲、拉伸、折叠等形变状态下 难以保持优异的性能. 本文通过低维度结构策略构筑了基于 $\mathrm{CsPBr}_{3}$ 纳米片和 $\mathrm{ZnO}$ 纳米线的柔性光电探测器. 得益于一维纳米 线和二维纳米片的高柔性, 所构筑的光电探测器在各种应力下表 现出优异的工作稳定性. 例如, 在弯曲 1000 次之后, 器件的性能没 有明显变化. 此外, 由于 $\mathrm{ZnO}$ 和 $\mathrm{CsPbBr}_{3}$ 自身的光吸收特性, 所构筑 的柔性光电探测器展现出宽光谱光电响应能力(涵盖紫外和可见波 段). 在紫外和可见区域的峰值响应度分别为 3.10 和 $0.97 \mathrm{~A} \mathrm{~W}^{-1}$, 其 相应的探测率分别为 $5.57 \times 10^{12}$ 和 $1.71 \times 10^{12}$ Jones. 本文针对柔性、 高性能集成光电探测器提出的维度构筑策略, 在未来智能、可穿 戴光电子器件领域有着巨大的应用前景. 